\title{
Les Problematiques Éthiques et Esthétiques à l'Exemple d'une Pratique Theatrale en Prison
}

\author{
Marie Urban \\ Aix-Marseille Université, France. \\ Universität Hildesheim, Allemagne. \\ Email: marie.urban.mrs@gmail.com
}

\section{Résumé}

La pratique du théâtre en prison pose la question de la participation de personnes non professionnelles dans des projets artistiques. Cet article est l'occasion d'observer les liens entre les problématiques éthiques et esthétiques, qui sont au cœur des discours sur la participation, à partir de l'exemple très singulier de la production artistique en milieu carcéral du collectif indépendant berlinois aufBruch ${ }^{1}$ . Je souhaite pour cela revenir sur la démarche que j'ai expérimentée à plusieurs reprises avec le metteur en scène Adrian Figueroa, lors de la réalisation de projets de théâtre et de film avec des détenus dans les prisons de Plötzensee et de Tegel à Berlin. Le travail se base sur des ateliers d'écriture et des interviews réalisées avec les détenus. Je décris à cette occasion les stratégies de distanciation mises en place durant ces projets afin de développer des formes littéraires proches de l'autofiction, dans lesquels émerge une tension entre récit de vie et imaginaire. En mêlant une approche théorique et pratique, il s'agit, de discuter de l'écueil à éviter d'“ exposer » les détenus, de questionner d'une part la fonction sociale de l'art pouvant s'affirmer comme un espace agonistique, et, d'autre part, de souligner les aspects esthétiques de ces projets qui oscillent entre espace authentique et symbolique. On pourra à cette occasion se demander comment les corps enfermés dans un lieu "absolument différent» (Foucault) peuvent être projetés dans un autre espace.

Mots-clés

Pratique théâtrale. Théâtre en prison. Ateliers d'écriture. Interviews.
Resumo

A prática do teatro na prisão levanta a questão da participação de profissionais não profissionais em projetos artísticos. Este artigo é uma oportunidade para observar os vínculos entre questões éticas e estéticas, que estão no centro dos discursos sobre a participação, a partir do exemplo muito singular de produção artística nas prisões do coletivo independente de Berlim aufBruch. Para fazer isso, gostaria de voltar ao processo que experimentei várias vezes com o diretor Adrian Figueroa, durante a realização de projetos de teatro e cinema com prisioneiros nas prisões de Plötzensee e Tegel em Berlin. O trabalho é baseado em oficinas de redação e entrevistas com prisioneiros. Nessa oportunidade, descrevo as estratégias de distanciamento implementadas durante esses projetos, a fim de desenvolver formas literárias próximas à autoficção, em que surge uma tensão entre a vida e a narrativa imaginária. Ao combinar uma abordagem teórica e prática, trata-se de transpor o obstáculo de evitar "expor" os prisioneiros e questionar, por um lado, a função social da arte que pode se afirmar como um espaço agonístico e, por outro lado, enfatizar os aspectos estéticos desses projetos que oscilam entre o espaço autêntico e simbólico. Nesta ocasião, podemos nos perguntar como os corpos encerrados em um lugar "absolutamente diferente" (Foucault) podem ser projetados em outro espaço.

Palavras-chave

Prática teatral. Teatro em prisão. Oficinas de escrita. Entrevistas.

1 URL : http://www.gefaengnistheater.de/ 


\section{La question de la participation}

Le théâtre germanophone est marqué actuellement par un accroissement des pratiques documentaires qui se manifestent par des formes variées. On parle dans certains cas de « théâtre de recherche ${ }^{1}$, car des recherches parfois longues, créant une archive hétérogène, peuvent être au cœur du processus de la création théâtrale. II est difficile de décrire ces créations car elles s'emparent du réel de diverses façons et font par ailleurs preuve d'un " état d'esprit performatif ", pour reprendre l'expression de Joseph Danan (Danan, 2016, p.59). La position des artistes, qui deviennent des sociologues ou des journalistes d'investigation, change également. On remarque aussi l'augmentation de la participation de personnes éloignées du monde du théâtre dans des processus de création. Ces participants sont tour à tour désignés comme étant des « témoins », des «invités $»^{2}$, des « experts du quotidien ${ }^{3}$, des « interprètes non professionnels » ou encore des « chercheurs associés "4. Ces différents termes reflètent les différentes façons d'intégrer ces personnes. Néanmoins, il

\footnotetext{
1 Le terme " théâtre de recherche " est une traduction du terme allemand "Recherchetheater " qui est de plus en plus employé par la critique mais aussi par des praticiens et des dramaturges: du 19 au 21 novembre 2015 ont eu lieu les « 1. Recherchetheatertage » (premières journées consacrées au théâtre de recherche » au Deutsches Theater de Berlin.
}

2 C'est le cas du groupe She She Pop lorsqu'elles invitent par exemple leurs propres pères à se mettre en scène avec elles dans la pièce Testament.

3 Traduction du terme « Experten des Alltags », introduit par la pratique de Rimini Protokoll mais qui est aujourd'hui employé plus largement.

4 Le psychologue Günter Mey a proposé durant les premiers "Recherchethatertage " (évoqué ci-dessus) de privilégier le mot " Mit-Forschenden » ce qui pourrait se traduire par « chercheur associé ou co-chercheur » plutôt que celui de « témoins » pour qualifier les participants intégrés au processus de création. s'agit souvent de réflexions collectives sur des phénomènes sociaux qui sont dévoilés ensuite sur scène. Les événements politiques récents, en particulier suite à l'augmentation conséquente du nombre de demandeurs d'asile en Allemagne depuis 2015, ont par ailleurs participé à l'émergence de nombreuses pièces ou performances dans lesquelles des réfugiés se trouvent mis en scène. Difficile de déterminer les fondements de ces démarches sans les observer au cas par cas, mais la complexité est posée: les motivations oscillent entre souhait d'une prise de conscience citoyenne, le désir de créer du lien social, ou encore le besoin de dénoncer des situations injustes. Ces pièces sont parfois à la frontière de l'activisme et du théâtre politique, mais il est également légitime de se demander s'il n'y a pas dans certains cas un phénomène de mode. En tous les cas, ce phénomène est très marqué dans l'espace germanophone et de nombreux théâtres allemands s'interrogent de façon croissante sur I'utilité du théâtre, son rôle politique, sa place dans la société. En France, la participation de « personnes réelles » et plus particulièrement de réfugiés est plus rare à la notable exception de la pièce 81, avenue Victor Hugo écrite par Olivier Coulon-Jablonka, Barbara Métais Chastanier et Camille Plagnet, qui met en scène des sans-papiers du 81 de l'avenue Victor Hugo à Aubervilliers, antenne désaffectée d'un Pôle emploi. Ce spectacle a eu beaucoup de résonance dans les médias et a contribué à la régularisation d'une majeure partie des membres du collectif qui s'y trouvait.

Les formes participatives concernent ici une implication de «personnes réelles » dès le processus de création et non la participation des spectateurs durant la représentation. L'accroissement de cette forme de participa- 
tion au théâtre semble être une façon de mettre à l'épreuve les questions de démocratie culturelle, mais aussi de se saisir directement des questions politiques brûlantes qui divisent actuellement les populations et les gouvernements européens. Ces pratiques laissent apparaître clairement des changements dans les modes de production créant de nouveaux espaces au sein desquels se développent des processus de transformation sociaux et esthétiques. Elles remettent en question les codes de la représentation, renouvellent les théâtralités, créent des formes entre fiction et réalité, génèrent du savoir et questionnent le rapport entre l'individu et le collectif. Mais elles posent également de nombreuses questions éthiques, qui sont renouvelées par l'apparition des réfugiés sur scène.

\section{Ethique et esthétique}

Dans son livre Artificell hells, Participatory Art and the Politics of Spectatorship (Bishop, 2012), Claire Bishop revient sur la complexité des implications éthiques de l'art participatif: elle observe les difficultés et divergences dans la façon d'évaluer des projets, qui se caractérisent non plus uniquement par leur valeur esthétique, mais aussi par leur impact social concret. Face à des projets ambigus, qui accordent parfois plus d'importance aux aspects humains, collaboratifs ou sociaux qu'aux aspects esthétiques, certains critiques ou commissaires d'exposition encouragent et privilégient les démarches qui ont des résultats concrets immédiats. Cet impact sur le réel implique entre autres l'effacement, au moins partiel, de la « marque » de l'artiste en tant qu'auteur: le jugement esthétique s'estompe au profit d'un jugement éthique. II faut souligner que l'analyse se porte sur des artistes provenant des arts visuels et sur un développement entamé dans les années 1990. On retiendra cependant la façon dont Claire Bishop réhabilite certaines questions esthétiques dans l'approche des œuvres participatives, qui risquent parfois de tomber dans la facilité d'un dialogue consensuel: "By contrast, I would argue that unease, discomfort or frustration - along with fear, contradiction, exhilaration and absurdity can be crucial to any work's artistic impact " (Bishop, 2012, p.26). Paul Ardenne met également en avant les développements de l'art éthique, dans la mesure où cet art aspirerait au tournant du XXlème siècle surtout à une forme d'amélioration sociale, ou politique. « $A$ ce registre, il arrive fréquemment que l'artiste " éthique », oubliant qu'il est un homme d'art, en vienne à sortir du territoire de la symbolisation et, se donnant en tout au contexte, qu'il se fasse ingénieur de bien-être ou de solidarité » (Ardenne, 2010, p.51-57).

Les liens entre les problématiques éthiques et esthétiques sont particulièrement complexes dans toutes les démarches qui impliquent des personnes « réelles », notamment lorsque ces dernières sont sur scène sans être formées au métier de comédien. Je souhaite envisager ces problématiques à partir de l'exemple très singulier de la production théâtrale en milieu carcéral et plus particulièrement à travers l'exemple de certains projets réalisés par aufBruch à Berlin. Après avoir présenté la démarche d'aufBruch, je décrirai la pratique que j'ai expérimentée à plusieurs reprises en tant qu'auteur et dramaturge avec le metteur en scène Adrian Figueroa dans différentes prisons de Berlin.

Même si elles sont insuffisantes, notons tout d'abord qu'il existe de nombreuses initiatives et 
pratiques artistiques en prison qui s'inscrivent dans des contextes très disparates. Sans faire un état des lieux des politiques culturelles envers les personnes placées sous main de la justice, il faut souligner les différences considérables entre les pays, mais aussi au sein même d'un pays. Les projets artistiques en prison dépendent en effet partiellement de décisions prises à un niveau plus local par les collectivités territoriales et elles sont souvent le fruit d'initiatives singulières provenant d'associations. Dans le système fédéral allemand, une partie du cadre législatif en matière de culture est fixé au niveau des Länder. De plus, la direction des institutions pénitentiaires n'encourage pas de la même façon les pratiques artistiques en prison et les intervenants doivent s'adapter à des fonctionnements différents d'une prison à l'autre, par exemple au niveau du rythme des détenus et du déroulement de leurs journées ${ }^{5}$.

L'art en milieu carcéral s'inscrit dans un contexte très spécifique qui consiste à s'adresser à des personnes tenues à l'écart de la société. L'amélioration progressive de l'accès à la culture en prison, a été possible par la reconnaissance de l'art comme contribuant à la construction de la personne. Jean-Louis Bernard souligne au sujet de la création artistique et de la dynamique d'insertion que

|« on est à chaque fois dans un processus de travail où à travers l'affrontement aux mots, aux matériaux, à son corps il s'agit bien de s'affronter d'abord à soi-même et aux autres. II s'agit là d'un vrai travail dans lequel les participants ont le sentiment qu'en créant un objet, un spectacle ou un livre, ils se construisent eux-mêmes » (Bernard, 2001, p.10).

5 Dans un entretien réalisé avec Sybille Arndt, directrice de production d'aufBruch, le 4 mars 2017, celle-ci témoigne en détail de ces différences d'une prison à l'autre et de la façon dont les répétitions doivent s'adapter à chaque situation spécifique et au règlement intérieur de chaque prison.
Beaucoup d'initiatives envers des publics détenus s'inscrivent dans la tradition d'un art social ou sont perçues comme tel, et d'autres, telle l'art thérapie, développent des pratiques à la croisée de plusieurs disciplines. Outre la question de la légitimité esthétique de ces pratiques artistiques, dont l'objectif premier serait l'impact social ou celui de l'insertion, Patrick Champagne s'interroge sur la fonction de l'artiste dans ce contexte, devenu « la figure dévalorisée de l'artiste engagé » (Champagne, 2001, p.38) ou endossant un « rôle de catalyseur et de révélateur des problèmes de société» (Ibid, p.38). La pratique des arts en prison étant toujours très singulière, il paraît pertinent d'observer des exemples précis.

Le travail d'aufBruch ne s'inscrit pas dans la tradition d'une démarche socioculturelle. L'exigence artistique est placée avant l'impact social, ce qui implique un engagement important de la part des détenus. On peut émettre I'hypothèse que cette démarche permettrait d'avoir une plus grande influence sur le développement personnel des détenus durant leur incarcération, qu'une pratique artistique occasionnelle moins engageante. Dans un article publié dans Le Monde datant de 1997, Jean-Louis Perrier notait déjà que certaines pratiques artistiques en milieu carcéral osent "prendre des risques authentiques en terme artistiques » et ont « une aptitude à élargir la problématique socioculturelle de base, voire à s'en différencier ». Le metteur en scène JeanPierre Chrétien-Goni préfère observer les esthétiques créées par ces pratiques politiques de l'art, plutôt que d'y voir un « art de la difficulté » (Chrétien-Goni, 2007). Par ailleurs, les formes évoquées plus haut, où des interprètes non professionnels sont mis en scène dans des théâtres, doivent, elles aussi, être examinées 
avec un regard critique par rapport à la qualité esthétique des spectacles. Ainsi, peu importe le lieu de la représentation, les questions éthiques et esthétiques sont indissociables.

\section{La pratique d'aufBruch}

Le théâtre en prison tel que le pratique aufBruch depuis 1997 (tout d'abord sous la direction de Roland Brus et Holger Syrbe) dans différentes prisons berlinoises repose sur une forme participative. La façon dont les projets sont structurés aujourd'hui est le fruit d'un perfectionnement progressif et raisonné des pratiques. Les membres permanents de l'équipe, constituée depuis 2003 de Peter Atanassow (metteur en scène), Holger Syrbe (scénographie et gestion) et Sybille Arndt (direction de production), ont amélioré le processus à force d'expérience. Leur objectif est d'obtenir les meilleures conditions de travail possible ainsi qu'un résultat de qualité. Ils ont réussi à négocier la fréquence des répétitions, l'obtention d'un espace de travail fixe et de suffisamment de temps, sachant que les détenus peuvent rejoindre les répétitions en règle générale après leur travail en milieu d'après-midi. Ils ont constaté par ailleurs l'importance de renforcer la cohésion du groupe ainsi que la responsabilité de chaque participant face au rôle qu'il a à jouer dans le projet. Les pièces de théâtre réalisées par aufBruch impliquent la participation de détenus volontaires, qui, après en moyenne sept semaines de répétitions, quatre jours par semaines et trois ou quatre heures par jour, deviennent les interprètes de pièces de théâtre. Les représentations se déroulent ensuite généralement sur deux à trois semaines à raison de trois représentations par semaine et sont ou- vertes à un public provenant de l'extérieur.

Les conditions de travail recherchées par aufBruch s'apparentent grandement à d'autres productions de théâtre et de performance dans la scène artistique indépendante allemande. aufBruch effectue d'ailleurs, comme tout autre groupe indépendant, des demandes de subventions par projet, auprès du sénat de Berlin ou auprès de fonds accordés aux arts de la scène ${ }^{6}$. Cette possibilité d'ouvrir l'enceinte de la prison à un large public - sous certaines conditions $^{7}$ - constitue une des singularités du travail d'aufBruch en comparaison avec les difficultés rencontrées par d'autres initiatives en Allemagne ou en Europe à faire de même. Bien que la présentation au public de manifestations artistiques au sein de prisons allemandes ne soit pas complètement inédite, Sybille Arndt explique que la particularité d'aufBruch réside dans la régularité des représentations, la professionnalisation du travail et l'accès aux manifestations ${ }^{8}$. Ceci est possible aujourd'hui grâce à de longues années de coopérations avec les institutions carcérales de Berlin et la confiance mutuelle qui s'est élaborée ${ }^{9}$. Enfin, cette démarche se distingue de beaucoup d'autres par le fait qu'elle accorde la priorité à l'exigence artistique avant le développement personnel des

\footnotetext{
6 Depuis peu, aufBruch est aussi soutenu par des fonds pour la kulturelle Bildung, c'est-à-dire pour la formation et l'éducation culturelle.

7 Les institutions pénitentiaires examinent la liste des spectateurs cinq jours avant la représentation : ils refusent l'entrée d'une personne lorsqu'ils soupçonnent que celle-ci souhaite entrer en prison pour un autre intérêt que la représentation théâtrale, comme par exemple pour faire du trafic ou rendre visite à un détenu. Les anciens détenus n'ont par ailleurs pas non plus le droit de pénétrer dans l'enceinte de la prison pendant six mois après leur sortie.
}

8 ARNDT, Sybille, 2017. Directrice de production d'aufBruch. Interview réalisée le 4 mars 2017, Berlin.

9 Les membres d'aufBruch arrivent dorénavant à faire valoir la reconnaissance et l'importance de leur travail malgré les changements de direction dans les différentes institutions. 
détenus. En tous les cas, ce travail ne rentre pas dans des plans officiels de réinsertion du système carcéral. aufBruch n'a pas non plus une démarche thérapeutique. La motivation principale pour tous les membres qui interviennent en prison (qu'il s'agisse des membres permanents ou des intervenants ponctuels) est de croire à la singularité, à la pertinence et à la qualité esthétique de ce théâtre joué par des détenus. Le collectif a conscience qu'il y a sans aucun doute une évolution personnelle positive pour les participants, devant développer esprit d'équipe et sens des responsabilités, mais celle-ci n'est ni recherchée explicitement, ni évaluée.

A l'aune des questions éthiques introduites plus haut, il serait possible de voir a contrario une forme d'instrumentalisation dans cette démarche. Les membres permanents d'aufBruch travaillent tout au long de l'année dans toutes les prisons de Berlin. Ils sont donc dépendants personnellement de ce travail, mais c'est également pour cette raison que la qualité du résultat est essentielle. II faut aussi insister sur le fait qu'un des objectifs d'aufBruch est de permettre une rencontre entre le public et les détenus, brisant ainsi l'hermétisme habituel de la prison. Les spectateurs sont amenés à remettre en question d'éventuels préjugés par rapport aux prisonniers: la qualité de la représentation et le fait que les détenus se sentent bien dans leur rôle sont pour cela essentiels. Jens Roselt observe ce renversement dans une mise en scène de Peter Atanassow en 2004 à la prison de Tegel, où les prisonniers observent à leur tour les spectateurs. Dans sa description de la pièce, il souligne le plaisir que prennent les détenus et la confiance qu'ils ont dans leur jeu (Roselt, 2006). De plus, les spectateurs et les détenus sont habituellement invi- tés à échanger durant une heure après la pièce : les prisonniers peuvent à cette occasion aller à la rencontre des spectateurs. Cette tradition permet ainsi de réduire le regard « voyeur » que peuvent avoir certains spectateurs.

\section{Description d'une pratique artistique spécifique}

Depuis 2014, j'ai collaboré avec aufBruch dans différentes prisons de Berlin à l'élaboration de deux pièces de théâtre, à des ateliers d'écriture et à un film qui est en cours de réalisation. Je travaille principalement en tant qu'auteur et dramaturge avec le metteur en scène Adrian Figueroa. Nous avons développé une pratique qui correspond à un théâtre de création, réalisé à partir de récits de prisonniers et mêlé à d'autres sources littéraires. Nous avons traité de thématiques telles que la représentation de soi, la mémoire, le rapport au temps en prison, ou encore la relation entre l'intérieur et l'extérieur.

La mise en scène d'interprètes non professionnels coïncide avec une recherche d'authenticité de plus en plus marquée sur les scènes contemporaines. Annemarie Matzke souligne qu'actuellement, l'omniprésence de la notion d'authenticité s'accompagne par ailleurs de doutes, voire d'un scepticisme partagé par toutes les disciplines. Elle relativise également le rapport entre authenticité et espace théâtral: « L'authenticité sur la scène est toujours l'effet d'une construction » (Matzke, 2006) ${ }^{10}$. C'est avec ce paradoxe que nous souhaitons explicitement jouer. Dans le cas présenté ici, le lieu de la représentation n'est pas

10 Librement traduit par nous. Version originale: « Authentizität auf der Bühne ist immer Effekt einer Konstruktion ». 
un théâtre, mais un lieu réel, fortement chargé d'autorité et de procédures. Paradoxalement, l'espace de la scène au sein de la prison est un espace de liberté et de possibles fictions. En travaillant avec les biographies des détenus, nous recherchons un ancrage supplémentaire dans le réel: celui de parcours de vie souvent intenses, fortement bousculés et l'expérience partagée de la détention. Le travail oscille entre authenticité et distance avec les matériaux récoltés. L'enjeu majeur est de mettre en scène des éléments biographiques partiellement fictionnalisés dans un cadre esthétique pertinent, sans exposer les détenus. Différentes stratégies de distanciation par rapport aux récits des détenus sont mises à l'œuvre au cours des étapes du processus. Le devenir partiellement fictionnel des récits réels constitue à la fois un élément esthétique fort du travail, tout en permettant un positionnement sur les questions éthiques. C'est finalement l'ambiguïté créée sur la véracité des propos, textes et témoignages entendus qui garantit en partie que les détenus soient «protégés ».

Concernant le processus, nous avons débuté les deux projets de théâtre par un atelier d'écriture ${ }^{11}$. Différents exercices d'écriture accompagnés de consignes amènent les participants à activer des éléments biographiques, des lieux ou personnes familiers, des situations vécues. Cependant, nous les encourageons aussi à exagérer ou à imaginer. Un exercice simple consiste par exemple à demander aux détenus de décrire ce qu'ils voient par la fenêtre: certains ont fait une description très précise, d'autres ont décrit ce dont ils rêvent

11 II s'agissait d'une part de la pièce Briefe an meine Katze Bébert en 2014 et d'autre part de la pièce Zeit vergeht. Warten en 2015, toutes deux réalisées dans la prison de Plötzensee à Berlin. J'ai réalisé ces deux ateliers d'écriture avec la romancière Mariana Leky. en regardant par la fenêtre, un autre encore a évoqué les avions qu'il voit dans le ciel et dans lesquels il s'imagine quitter l'Allemagne. Outre la thématique de leur quotidien dans l'univers carcéral, nous avons eu différentes occasions de travailler sur le souvenir, en leur demandant par exemple de décrire précisément la rue de leur enfance. Les contraintes formelles donnent un cadre précis au sein duquel une force poétique peut s'échapper: les listes inspirées d'Espèces d'espaces de George Perec ou encore de son livre Je me souviens ont été d'importantes sources d'inspirations. Nous travaillons aussi avec des questionnaires qui nous permettent de découvrir des anecdotes ou éléments de leurs biographies, pouvant être atypiques ou ayant un fort potentiel narratif. Cela nous permet ensuite de leur demander d'approfondir une histoire ou un événement en particulier. II faut toutefois souligner que les niveaux de lecture et d'écriture de l'allemand des détenus sont très disparates. Nous avons donc préféré dans certains cas retranscrire des interviews dont des fragments peuvent ensuite être intégrés au montage. De manière générale, nous avons la plupart du temps utilisé des fragments comme matière textuelle des pièces finies. Dans certains cas, il nous a semblé plus pertinent de maintenir la chronologie de leur parcours, afin de saisir l'enchaînement de certains événements ou de certaines formes d'engrenage (par rapport à la consommation ou à la vente de drogues par exemple). L'écriture et les discussions nous amènent parfois à connaître la raison de leur incarcération, mais il est important de préciser ici que nous ne leur posons jamais explicitement la question et que nous n'avons pas accès à leurs dossiers.

Les textes produits par les détenus s'approchent du genre littéraire de l' « autofiction », 
c'est-à-dire que même si l'auteur, le narrateur et le personnage ne font qu'un, « l'écriture est créatrice de fiction » (Gasparini, 2008, p.205). De plus, de nombreuses théories en sciences sociales démontrent que " la vérité biographique n'est pas accessible ${ }^{12}$. La construction de l'identité est subjective et l'influence des processus de socialisations intersubjectifs dans cette construction doit être prise en compte (Berger, Luckmann, 1966). Afin de mettre en exergue les composantes narratives qui accompagnent tout récit de vie, il faut en outre revenir plus en détail sur le concept d' " identité narrative » tel que le développe Paul Ricoeur (Ricoeur, 1990). Les liens que ce dernier établit entre récit et fiction interviennent à différents niveaux. Dans le cas de notre travail avec les histoires racontées ou écrites par les détenus, nous retiendrons plus particulièrement le potentiel narratif pour le devenir scénique des récits de vie. Insistons sur le fait que l'Homme articule ses expériences sous forme d'histoires, puis le récit et l'écriture permettent de faire entrer pleinement le vécu dans la fiction. Cette « mise en intrigue » de soi-même par le récit contribue également à une meilleure compréhension de soi. Concernant le théâtre en prison,l'enjeu est donc double : la construction de soi ainsi que la production d'une parole collective au sein d'un environnement dont le premier principe est celui de l'isolement (Foucault, 1975, p.274).

Je souhaite ici faire une digression en soulignant que certains détenus rencontrés lors de nos projets dans les prisons berlinoises mènent une pratique régulière de l'écriture sous forme de journaux intimes, recueils de poèmes ou

12 L'ouvrage collectif qui s'intitule « Die biographische Wahrheit ist nicht zu haben » publié par Klaus Jürgen Bruder a pour titre cette citation de Freud écrite dans une lettre adressée à Zweig en 1939. encore de romans. Philippe Lejeune (Lejeune, 1998) fait remarquer que le manque d'intimité en prison, rend presque impossible toute forme d'écriture et plus particulièrement l'écriture autobiographique. Chris-Bär Templiner, incarcéré dans la prison de Tegel à Berlin, témoigne ${ }^{13}$ pourtant d'une expérience contraire. Il écrit autant de façon autobiographique que fictionnelle (prose et poésie) et il a tenu pendant longtemps un journal intime. Et il lit en moyenne deux livres par semaine. Helmut Kühn ${ }^{14}$ (également incarcéré à Tegel) fait état d'une pratique similaire, pouvant, lui aussi, écrire complètement librement. Ce dernier fait preuve d'ailleurs d'une extrême productivité: il dit avoir écrit cinq livres depuis 2012, ce qui a par contre réduit sa pratique de lecteur. L'analyse de Philippe Lejeune repose sur les prisons françaises. Les conditions d'incarcération - comme le fait d'être seul ou pas dans une cellule - influencent sans aucun doute la capacité des détenus à écrire et aussi à participer assidûment à d'autres activités artistiques. Sans rentrer dans le détail, les problèmes sanitaires et de surpopulation qui rendent l'écriture en prison plus difficile, sont plus importants en France qu'en Allemagne.

Il a été intéressant de remarquer que nous ne pouvions pas différencier le vrai du faux dans les récits des détenus : cet élément devait donc précisément jouer un rôle central dans notre démarche. Le processus éthique et esthétique de ce travail en prison se base sur cette ambiguiité pour déjouer les attentes des spectateurs curieux, voire avides de « confessions

13 Chris-Bär Templiner incarcéré depuis plus de trente ans, témoigne dans une lettre qu'il m'adresse le 5 mars 2017 de ses pratiques d'écritures et de lectures en réponse à un questionnaire que je lui avais donné au sujet de sa pratique de l'écriture et de la lecture.

14 Helmut Kühn a répondu au même questionnaire mentionné ci-dessus dans une lettre qu'il m'adresse le 13 mars 2017. 
impudiques ». Louis Patrick Leroux décrit ce phénomène comme suit: "La confession dite impudique, est souvent liée à un traumatisme, à un aveu de la part du comédien: homosexualité, maladie, comportement qu'il se reproche. II s'agit d'un témoignage intime permettant à l'acteur/auteur d'exorciser une problématique qui l'accablait » (Leroux, 2004, p.79). II souligne ensuite que " la confession impudique gêne une part du public par sa nature exhibitionniste et attise l'intérêt des autres spectateurs » (lbid, p.79). Impossible d'éviter complètement la recherche de sensationnel de certains membres de l'audience et la confession (impudique) de certains détenus, mais différentes stratégies permettent d'atténuer ce rapport, car il semble à juste titre problématique au niveau éthique et esthétique. Notons de plus que le passage à la scène permet une forme de distanciation supplémentaire par rapport aux sources référentielles.

Mais revenons aux différentes étapes du processus créatif. La matière collectée est assemblée, les textes sont condensés et coupés. Les matériaux choisis sont ensuite réintégrés dans un montage, c'est-à-dire dans « une nouvelle chaîne de signification » (Piemme, 2011). J'ai également introduit des textes littéraires empruntés, entre autres, à Ferdinand Céline, Virginie Despentes, Max Frisch ou encore Sarah Kane aux textes des détenus. Ceci permettait d'accentuer la confusion, décrite plus haut, concernant la véracité des propos des détenus.

La pièce constituée d'un collage de textes évolue encore au fur et à mesure des répétitions et les textes mémorisés par les détenus sont mis à l'épreuve de la théâtralité de la scène. Une équipe constituée d'intervenants extérieurs à l'institution pénitentiaire, qui sont des artistes professionnels, participent à la deuxième phase du travail: durant les répétitions, ils créent la musique, les costumes ou encore la vidéo (qui joua un rôle important dans les deux mises en scène réalisées à Plötzensee). II ne s'agit pas ici de décrire en détail ces mises en scène, mais de préciser que l'approche esthétique n'est pas brute ou minimaliste. Elle accorde au contraire de l'importance aux aspects visuels et sonores des spectacles. Dans la pièce Zeit vergeht. Warten (2015), une partie de la scénographie était constituée d'un fond vert devant lequel les interprètes venaient parfois parler face à une caméra, tandis que l'image était retransmise ailleurs dans l'espace scénique avec des projections vidéos. Ce procédé de dédoublement permettait de voir la projection de l'interprète dans un autre lieu, dans un autre contexte, et simultanément sa présence réelle sur la scène. Ajoutons à cela que certains procédés performatifs contribuèrent dans les deux mises en scène à briser un peu plus les barrières entre «le dehors et le dedans ». Dans la pièce Briefe an meine katze Bébert (2014), une caméra était installée dans une pièce adjacente à la salle de la représentation. Lors d'une scène, quelques spectateurs volontaires étaient invités à aller fumer une cigarette avec certains détenus dans cet espace, tandis que l'action qui s'y déroulait était retransmise en live sur la scène principale. Autre originalité: dans les deux spectacles (de 2014 et 2015), un détenu cuisinait sur scène pendant toute la durée de la représentation et le repas était ensuite servi au public, invité à rester après la représentation.

Ainsi, l'authenticité de certains textes se mêlait à la théâtralité des autres éléments de la représentation ainsi qu'à certains aspects performatifs. Le passage à la scène décrit briè- 
vement ici correspond donc à une étape importante, où les récits écrits par les détenus font alors partie d'un dispositif théâtral et d'un processus créatif collectif.

L'essentiel de ce travail consiste d'une part à utiliser ce qu'ils sont, pour être plus précis, la façon dont ils se perçoivent, mais aussi les personnages qu'ils se sont crées, ce qu'ils souhaiteraient être ou encore les mondes fantastiques ou imaginaires qu'ils cultivent parfois. D'autre part, il s'agit aussi, à travers le montage des textes et la mise en scène, de ce que nous projetons sur eux, de la façon dont nous les percevons. Dans cet écart de perceptions apparaît une tension qui est sans cesse interrogée et réajustée puisque ce type de création n'est jamais figé. Ce procédé d'allers-retours se démarque de nombreuses démarches autobiographiques au théâtre, comme celles où l'auteur lui-même se met en scène. Tel que nous l'avons abordé plus haut, les pratiques documentaires actuelles mettant en scène par exemple des « experts du quotidien » tout comme des réfugiés correspondent à des démarches toujours singulières, qui se distinguent fortement les unes des autres, dans la façon dont elles représentent la société et dans leurs implications politiques. Le théâtre en prison s'inscrit dans une tradition consistant à donner la parole à ceux qui ne l'ont pas, à refléter la diversité de la société et à encourager l'émancipation de chacun par le langage et l'art tels qu'Augusto Boal et Armand Gatti l'expérimentèrent (malgré leurs différences). Le déplacement du lieu théâtral renforce également I'utilité du théâtre quant à son ancrage dans le réel et le présent en affirmant son rôle en tant qu'espace agonistique.

La façon de travailler, d'impliquer et d'inclure les participants à un projet, est essentielle au regard des problématiques éthiques qui se posent avec la mise en scène d'interprètes non professionnels. Ces expériences menées en prison révèlent l'importance de ces questions, car le contexte de l'emprisonnement renforce la vulnérabilité des participants. Le risque de les exposer est d'autant plus fort qu'il s'agit d'un cadre disciplinaire où un pouvoir s'effectue sur les corps et les esprits, pour reprendre Foucault:

« La prison doit être un microcosme d'une société parfaite où les individus sont isolés dans leur existence morale, mais où leur réunion s'effectue dans un encadrement hiérarchique strict, sans relation latérale, la communication ne pouvant se faire que dans le sens de la verticale » (Foucault, 1975, p.276).

C'est pourquoi l'espace d'expression créé par ces projets, qui donnent librement la parole aux détenus en communication avec des intervenants extérieurs au système judiciaire et carcéral (sans objectif thérapeutique, pédagogique ou de réinsertion à proprement parler), semble être extrêmement précieux. II est très important de préciser aux détenus que leur participation au projet ne leur permettra pas obligatoirement de réduction de peine. Ne pas faire de fausses promesses: voilà qui semble essentiel, d'autant plus dans le contexte particulier de l'univers carcéral et de la privation de liberté. De manière générale, cela devrait être la règle dans tous les cas où des « personnes réelles » sont impliquées dans un projet artistique. Par ailleurs, le fait que les amateurs n'ont pas forcément conscience de l'image qu'ils renvoient est un problème fréquemment soulevé au sujet des pièces qui mettent en scène des « personnes réelles ». Cette question renforce la responsabilité de l'équipe artistique qui porte le projet. Notre position par rapport à cela (qui est une réponse possible parmi d'autres) 
est celle de créer un échange, qui s'approche de la démarche des «images négociées » du photographe Michel Séméniako qui réalise des portraits en concertation avec ses sujets. Le film Anderswo qui est en cours de réalisation s'inspire de cette pratique où l'art intervient sur le réel et où le réel intervient sur les images artistiques.

Le cadre spatio-temporel constitue la spécificité de la représentation théâtrale. La dissolution du temps et l'espace confiné de la prison, sont au centre de l'expérience carcérale. Le théâtre et la prison sont des lieux « absolument différents ", des « lieux hétérotopiques » (Foucault, 2009, (1966)). Des lieux ouverts ou fermés mais « qui les isole par rapport à l'espace environnant » (Foucault, 2009, (1966) p.32). Quelle place peut alors avoir le théâtre en prison? « II se trouve que les hétérotopies sont liées le plus souvent à des découpages singuliers du temps » (Ibid, p.30). Et comment appréhender le rapport au corps, lorsque ces espaces-temps de l'incarcération et de la représentation se mêlent? « II [le corps] est au cœur du monde ce petit noyau utopique à partir duquel je rêve, je parle, j'avance, j'imagine, je perçois les choses en leur place et je les nie aussi par le pouvoir indéfini des utopies que j'imagine » (Ibid, p.18). $\mathrm{Au}$ contact de personnes enfermées parfois pour de longues peines, on se rend compte du rôle vital que peut jouer dans leur quotidien la musique, la lecture ou l'écriture. Le corps ne s'échappe symboliquement que par l'imagination. Les expériences théâtrales menées jusqu'à présent ainsi que le film en cours de réalisation nous ont permis de développer une façon de travailler dans l'univers carcéral (qui reste indissociable de son contexte de production en Allemagne, à Berlin et avec aufBruch): le fruit d'un échange qui oscille entre le témoi- gnage réel et la création d'images poétiques. "L'imagination dans ses vives actions, nous détache à la fois du passé et de la réalité 》 (Bachelard, 2009, (1957), p.16). Et pourtant, le réel des parcours de vie des détenus possède une force narrative incontestable, car leurs histoires sont marquées parfois par l'exil, la violence, la solitude, la fuite, la culpabilité ou encore tiraillés entre un rapport hédoniste à l'instant présent et raisonnable à l'avenir. Leurs récits et les images poétiques qu'offre la création artistique en prison semblent illustrer avec force que « le matérialisme de la fiction éprouve l'irréel de la réalité tandis que l'incroyable du réel ne trouve d'équivalent que dans la fiction » (Pic, 2010). Les images réelles et symboliques ainsi que l'ambigüité entre le réel et fictionnel laissent apparaître un espace poétique. Enfin, les formes de socialité créées à travers cette démarche (tout comme d'autre pratiques interventionnistes en art), associées aux problématiques éthiques transportées reflètent de nouvelles pratiques politiques en art, au sein desquels les aspects sociaux, esthétiques et symboliques sont indissociables.

References

ARDENNE, Paul, L'avenir éthique de l'art. Nouvelle revue d'esthétique, $2 / 2010$. $N^{\circ} 6$, (p. 51-57). [URL: http://www.cairn.info/revue-nouvelle-revue-d-esthetique-2010-2-page-51. htm], (page consultée le 3 mars 2017).

BACHELARD, Gaston. La poétique de l'espace. Paris: Presses universitaires de France, 2009 (1957). 
BERNARD, Jean-Louis. Introduction. In: BERNARD, Jean-Louis (coord). La création artistique et collective au cœur de la dynamique d'insertion.Budapest; Torino: I'Harmattan, 2001.

BISHOP, Claire. Artificell hells, Participatory Art and the Politics of Spectatorship.London, New York : Verso, 2012.

CHAMPAGNE, Patrick. Digression autour des fonctions de l'art. In : BERNARD, Jean-Louis (Coord.). La création artistique et collective au cœur de la dynamique d'insertion.Budapest ; Torino : I'Harmattan, 2001. (p.37-44).

CHRÉTIEN-GONI, Jean-Pierre. Artistes in extremis. In : Les hors-champs de l'art. Prisons, psychiatrie, quelles actions artistiques ? Cassandre/Horschamp, 2007. (p.187-196).

DANAN, Joseph. Entre théâtre et performance. Arles : Actes Sud, 2016.

FOUCAULT, Michel. Le corps utopique, les hétérotopies. France : Nouvelles Editions Lignes, 2009 (1966).

FOUCAULT, Michel. Surveiller et punir, Naissance de la prison. Paris : Gallimard, 1975.

GASPARINI, Philippe. Autofiction,Une aventure du langage. Paris : Seuil, 2008.

LEJEUNE, Philippe. Ecrire à l'ombre 1 et Ecrire à l'ombre 2. In: Pour l'autobiographie, Chroniques. Paris: Seuil, 1998. (p.104-112).

LEROUX, Louis Patrick. Théâtre autobiographique: quelques notions. In : La tentation biographique. Jeu: revue de théâtre, $n^{\circ} 111$, (2) 2004. Consultable en ligne: http://id.erudit. org/iderudit/25505ac, (page consulté le 14 avril 2017). (p.75-85).
MATZKE, Annemarie. Von echten Menschen und wahren Performern. In: FISCHER-LICHTE Erika, GRONAU, Barbara (Coord.), Wege der Wahrnehmung, Authentizität, Reflexivität und Aufmerksamkeit im zeitgenössischen Theater. Berlin: Theater der Zeit, Recherche 33, 2006. (p.39-47).

PERRIER, Jean-Louis. Le monde carcéral s'ouvre à la pratique des arts. Le Monde, Samedi 22 mars 1997.

PIC, Muriel. W.G. Sebald. Les patiences de la mémoire. In: CRIQUI, Jean-Pierre (Coord.). L'image document, entre réalité et fiction. $\mathrm{Pa}$ ris: Les carnets du bal \#1, Le Bal / Images en manœuvres éditions, 2010. (p.92-119).

PIEMME, Jean-Marie. Fiction toujours. In: PIEMME, Jean-Marie, LEMAIRE, Véronique (Coord.). Usages du « document », Les écritures théâtrales entre réel et fiction. Louvain-la-Neuve: Etudes théâtrales 50, 2011. (p.93).

ROSELT, Jens, Die Arbeit am Nicht-Perfekten. In: FISCHER_LICHTE, Erika, GRONAU, Barbara (Coord.). Wege der Wahrnehmung: Authentizität, Reflexivität und Aufmerksamkeit im zeitgenössichen Theater. Berlin: Theater der Zeit, Recherche 33, 2006. (p.28-38).

RICOEUR, Paul. Soi-même comme un autre, Paris: Le Seuil, 1990.

Recebido em 18/09/2017 Aprovado em 16/11/2017 